STED nanoscopy with wavelengths at the emission maximum

This content has been downloaded from IOPscience. Please scroll down to see the full text. 2016 J. Phys. D: Appl. Phys. 49365102

(http://iopscience.iop.org/0022-3727/49/36/365102)

View the table of contents for this issue, or go to the journal homepage for more

Download details:

IP Address: 134.76.223.157

This content was downloaded on 19/09/2016 at 14:58

Please note that terms and conditions apply.

You may also be interested in:

Dynamic far-field fluorescence nanoscopy

V Westphal, M A Lauterbach, A Di Nicola et al.

Sub-Abbe resolution: from STED microscopy to STED lithography

Thomas A Klar, Richard Wollhofen and Jaroslaw Jacak

Phase filter enhanced STED-4Pi fluorescence microscopy: theory and experiment

M Dyba, J Keller and S W Hell

Exploring single-molecule dynamics with fluorescence nanoscopy

Christian Ringemann, Ben Harke, Claas von Middendorff et al.

Fluorescent dyes with large Stokes shifts for super-resolution optical microscopy of biological objects: a review

Maksim V Sednev, Vladimir N Belov and Stefan W Hell

Stimulated emission depletion microscopy on lithographic nanostructures

Volker Westphal, Jens Seeger, Tim Salditt et al.

The 2015 super-resolution microscopy roadmap

Stefan W Hell, Steffen J Sahl, Mark Bates et al. 


\title{
STED nanoscopy with wavelengths at the emission maximum
}

\author{
Martín D Bordenave ${ }^{1,3}$, Francisco Balzarotti ${ }^{3}$, Fernando D Stefani ${ }^{1,2}$ \\ and Stefan W Hell ${ }^{3}$ \\ ${ }^{1}$ Centro de Investigaciones en Bionanociencas (CIBION), Consejo Nacional de Investigaciones \\ Científicas y Técnicas (CONICET), Godoy Cruz 2390, C1425FQD, Buenos Aires, Argentina \\ 2 Departamento de Física, Facultad de Ciencias Exactas y Naturales, Universidad de Buenos Aires, \\ Pabellón 1, Ciudad Universitaria, C1428EHA, Buenos Aires, Argentina \\ 3 Department of Nanobiophotonics, Max Planck Institute for Biophysical Chemistry, Göttingen, Germany \\ E-mail: shell@gwdg.de (SWH) and fernando.stefani@ cibion.conicet.gov.ar (FDS)
}

Received 16 May 2016, revised 22 June 2016

Accepted for publication 5 July 2016

Published 9 August 2016

\begin{abstract}
Commonly, in stimulated emission depletion (STED) fluorescence nanoscopy, light of a wavelength located at the red tail of the emission spectrum of the dye is used to shrink the effective fluorophore excitation volume and thus to obtain images with sub diffraction resolution. Here, we demonstrate that continuous wave (CW) STED nanoscopy is feasible using STED wavelengths located at the emission maximum, where the cross section for stimulated emission is up to 10 -fold larger than at the red tail. As a result, STED imaging becomes possible at equally lower STED beam power. Besides, fluorophores that have been considered inapplicable in certain wavelength constellations are thus becoming usable.
\end{abstract}

Keywords: fluorescence microscopy, superresolution, nanoscopy, resolution, acousto-optical devices

(Some figures may appear in colour only in the online journal)

\section{Introduction}

The family of methods called far-field fluorescence nanoscopy has brought fluorescence imaging into a new dimension. They keep virtually all the benefits of fluorescence microscopy while providing a resolution conceptually limited only by the size of the fluorescent label. The key enabling concept is the transient state transfer or switching of fluorophores between (at least) two distinguishable states, typically an emissive state (fluorescence 'on') and a non-emissive state (fluorescence 'off'). Fluorescence 'off' can refer to a fluorophore state that is truly unable to emit fluorescence photons, or to a state that emits in a spectral range not detected in the experiment. This on-off state transfer permits fluorophores to be told apart even if they are closer to each other than the distance set by the diffraction limit $[1,2]$.

There exist two families of far-field fluorescence nanoscopy methods [2]: (i) the methods with coordinate-stochastic readout usually implemented in wide-field configurations, and (ii) the scanning methods with coordinate-targeted readout such as STED and RESOLFT (reversible saturable optical fluorescence transition). In the targeted techniques, the on-off state switching is induced by a light pattern featuring intensity minima and maxima, such as a 'doughnut' or a pattern of standing waves. Within each family of methods an increasing number of physicochemical ways, i.e. state transitions, exist to effectively switch fluorophores on and off [3-7]. STED nanoscopy in particular is based on the off-switching of fluorophores by stimulated emission using a so called STED or depletion laser. The 'on' state is the first electronically excited fluorescent (singlet) state of a fluorophore whereas the 'off' state is the ground state.

Usually, the wavelength of the STED beam is carefully chosen to be at the far-red tail of the emission spectrum of the fluorophores used, so as to leave a comfortable spectral window for fluorescence detection between the excitation and STED wavelengths. However, this configuration makes STED rather inefficient because the stimulated emission cross section $\sigma_{\text {se }}$ follows practically the same spectral dependency as the emission $[8,9]$ : 


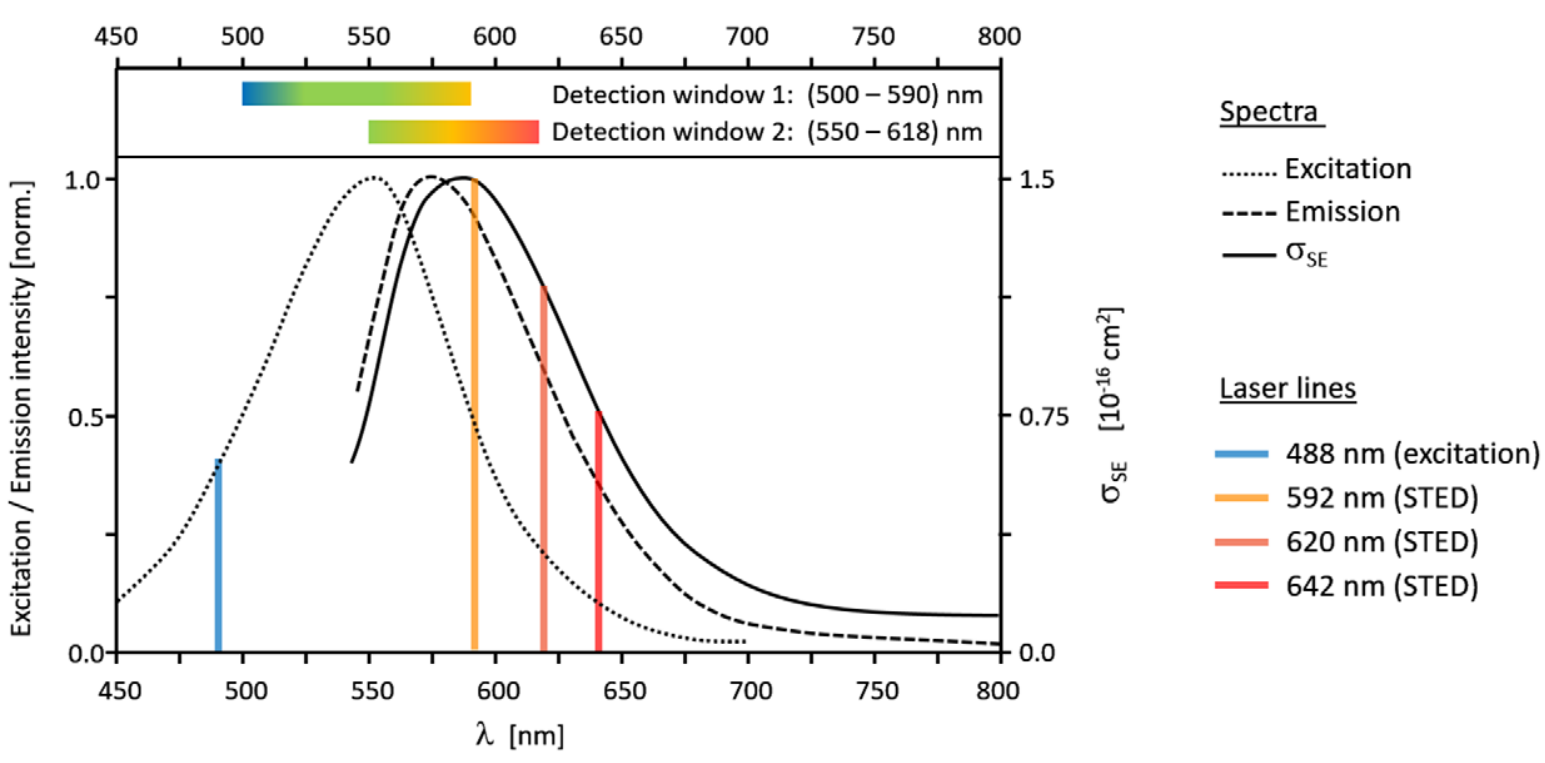

Figure 1. Normalized emission (excited at $488 \mathrm{~nm}$ ) and excitation (detected at $(710 \pm 10) \mathrm{nm})$ spectra of Nile Red in a poly(methyl methacrylate) film, together with the calculated stimulated emission cross section, using equation (1), $\phi=0.7$, and $\tau=(3 \pm 0.1) \mathrm{ns}$. Vertical lines indicate the wavelengths used in this work for fluorescence excitation $(488 \mathrm{~nm})$ and STED $(592 \mathrm{~nm}, 620 \mathrm{~nm}$ and $642 \mathrm{~nm})$.

$$
\sigma_{\mathrm{se}}(\lambda)=\frac{\lambda^{4} E(\lambda) \phi}{8 \pi c n^{2} \tau}
$$

where $E(\lambda)$ is the emission intensity as a function of the wavelength $(\lambda)$ normalized so that its integral equals one, $\phi$ is the quantum efficiency, $c$ is the speed of light in vacuum, $n$ is the refractive index and $\tau$ is the lifetime of the excited state.

In order to compensate for the small values of $\sigma_{\mathrm{se}}$, comparatively high power densities are needed for efficient STED, typically of about several tens of $\mathrm{MW} \mathrm{cm}^{-2}$, i.e. 1000-10000 fold what is needed for effective fluorophore excitation. These high levels of irradiance have raised concerns about phototoxicity $[10,11]$, and motivated the development of alternative coordinate-targeted super resolution approaches using photo-switchable fluorescent labels and lower intensities of light $[1,2,4,12-15]$. Yet, STED nanoscopy remains attractive because stimulated emission is a universal off-switching mechanism applicable in principle to any fluorophore, and has in fact been successfully applied to live-cell [16-21] and in vivo [22] imaging.

Therefore, the use of STED wavelengths closer to the emission maximum, but still at the long-wavelength tail, has been investigated and STED super-resolved images with lower intensities of the STED laser were obtained [23, 24]. Here, we further explore such a scheme demonstrating that STED nanoscopy is feasible using STED wavelengths across the complete emission spectrum of the fluorophore (figure 1). We used a multicolor continuous wave (CW) STED nanoscope based on acousto-optic wavelength selection, both for excitation and detection, which enables the proper comparison of super-resolved images obtained at different STED wavelengths tuned across the emission maximum of the fluorophore. For a given power density, shifting the $\mathrm{CW}$ STED wavelength toward the emission maximum leads to a higher resolution, in agreement with the expected increase in the stimulated emission cross section. We also find that, for a given power density, tuning the wavelength across the emission spectrum leads to a trade-off between resolution and overall signal.

\section{Materials and methods}

\subsection{Sample preparation}

The experiments were carried out on samples of $20 \mathrm{~nm}$ polystyrene beads dyed with the fluorophore Nile Red (Nile Red Fluospheres Invitrogen), dispersed on a glass substrate at a surface density of about one bead per $4 \mu \mathrm{m}^{2}$. Glass substrates (microscope slides \#1.5 Marienfeld) were cleaned by the following steps: (i) 10 min sonication in $0.2 \% \mathrm{v} / \mathrm{v}$ Hellmanex II in Milli-Q water, rinsing with Milli-Q water; (ii) 10 min sonication in ethanol; (iii) $10 \mathrm{~min}$ sonication in acetone; (iv) dried with $\mathrm{N}_{2}$ and finally cleaned using plasma. Then, a thin poly-L-lysine (Sigma-Aldrich) film was deposited onto the glass substrates by (i) immersing the cleaned glass substrates in a poly-L-lysine solution $0.1 \% \mathrm{w} / \mathrm{v}$ in water for $2 \mathrm{~min}$; (ii) rinsing them with abundant Milli-Q water and (iii) drying them with $\mathrm{N}_{2}$. The carboxylate-modified fluorescent beads, where diluted in Milli-Q water in four consecutives steps, until arriving to a concentration of $1: 230000$ of the original suspension. The diluted suspension was sonicated for $30 \mathrm{~min}$, centrifuged at $12000 \mathrm{rpm}$ for $2 \mathrm{~min}$. A drop of the supernatant was placed on the treated microscope slides for $30 \mathrm{~s}$, and then washed away with abundant Milli-Q water. The sample was then dried with $\mathrm{N}_{2}$ and mounted on a microscope coverslip with $5 \mu \mathrm{l}$ of mowiol mounting medium.

\subsection{Spectral properties of Nile Red}

In figure 1 the following spectra of Nile Red are shown: (i) normalized emission spectra obtained with an excitation 
(a)

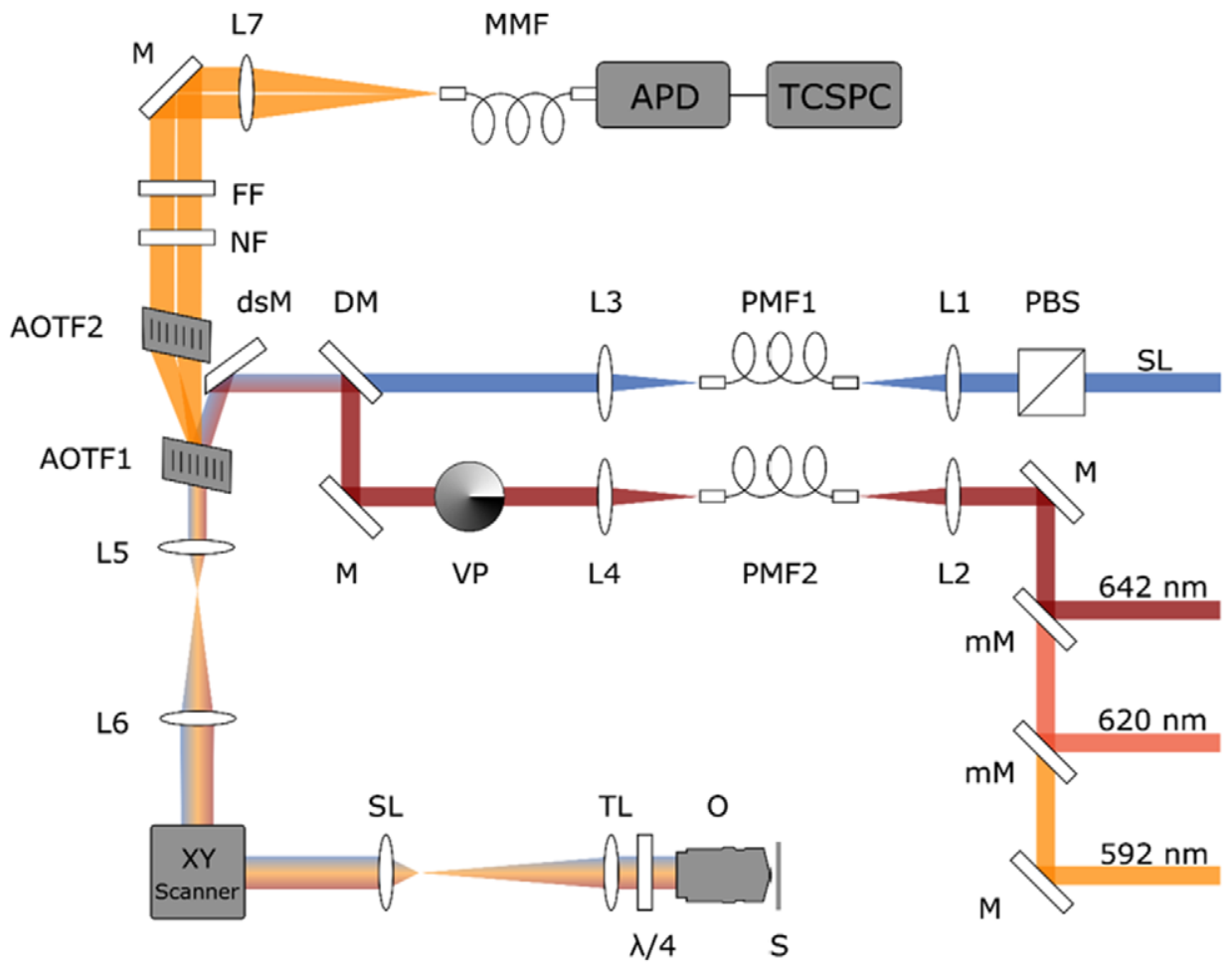

(b)

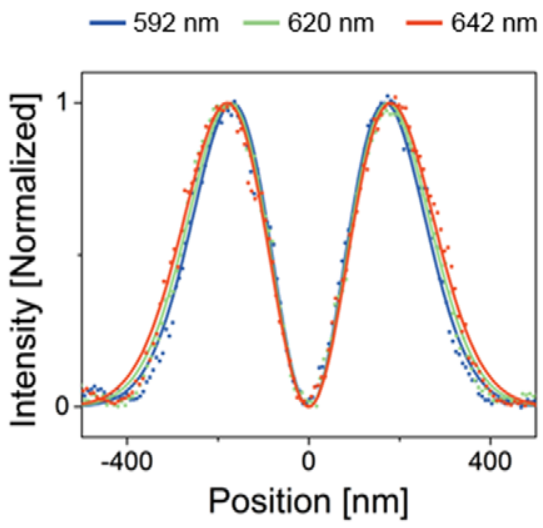

(c)
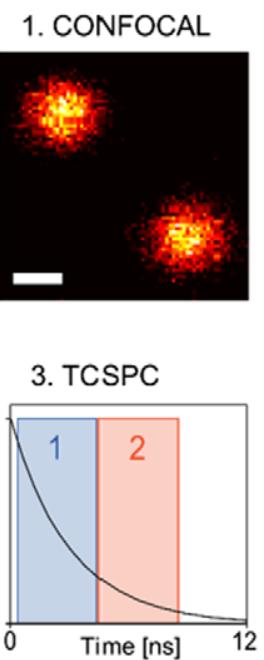

2. STED

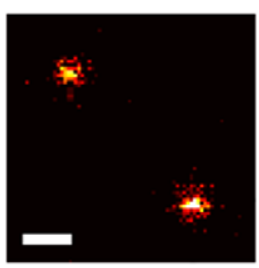

4. STED

reconstructed

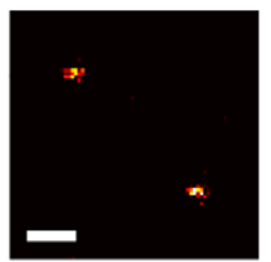

Figure 2. (a) Schematic representation of the multicolor CW STED nanoscope. SL: supercontinuum laser, PBS: polarizing beam splitter, M: mirror, L: lens, PMF: polarization maintaining single-mode fiber, mM: mirror in a magnetic mount, VP: vortex phase plate, DM: dichroic mirror, dsM: D-shaped mirror, AOTF: acousto optical tunable filter, SL: scanning lens, TL: tube lens, $\lambda / 4$ : $\lambda / 4$ waveplate, O: objective, S: sample, NF: notch filter, FF: fluorescence filter, MMF: multi-mode fiber, APD: avalanche photo diode, TCSPC: time correlated single photon counting device. (b) Signal profiles (dots) and fits (lines) corresponding to each of the doughnut-shaped STED focus. (c) Sequence of data acquisition with example images: (1) confocal image. (2) CW STED raw image. (3) Nile Red spontaneous emission decay and time windows used to subtract fluorescence background excited by the STED beam. (4) STED image with background subtracted. Scale bar is $200 \mathrm{~nm}$.

wavelength of $488 \mathrm{~nm}$; (ii) normalized excitation spectra detected at a wavelength of $(710 \pm 10) \mathrm{nm}$; and (iii) the wavelength dependent stimulated emission cross section calculated according to equation (1), with the experimentally measured excited state lifetime of $(3 \pm 0.1)$ ns and the maximum value of quantum efficiency reported for Nile Red $(\mathrm{QE}=0.7$; in pure dioxane) [25].

\subsection{Pulsed excitation, CW STED wavelengths}

Also in figure 1, the laser lines used for excitation $(488 \mathrm{~nm})$ and for STED $(592 \mathrm{~nm}, 620 \mathrm{~nm}$ and $642 \mathrm{~nm})$ are shown. The calculated stimulated emission cross sections corresponding to the three STED wavelengths are $\sigma_{\mathrm{se}}(592 \mathrm{~nm})=1.4 \times 10^{-16} \mathrm{~cm}^{2}$, $\sigma_{\mathrm{se}}(620 \mathrm{~nm})=1.1 \times 10^{-16} \mathrm{~cm}^{2}, \sigma_{\mathrm{se}}(642 \mathrm{~nm})=0.7 \times 10^{-16} \mathrm{~cm}^{2}$. 
(a)

CONFOCAL

\begin{tabular}{|c|c|c|c|c|c|c|}
\hline des & $5 \mathrm{~mW}$ & $15 \mathrm{~mW}$ & $25 \mathrm{~mW}$ & $40 \mathrm{~mW}$ & $65 \mathrm{~mW}$ & $110 \mathrm{~mW}$ \\
\hline $642 \mathrm{~nm}$ & No change & (5) & 7 & $\$$ & $\approx$ & 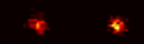 \\
\hline $620 \mathrm{~nm}$ & No change & 48 & Bद & 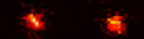 & s & 2 \\
\hline $592 \mathrm{~nm}$ & S3t & 6 & is & Low signal & Low signal & Low signal \\
\hline
\end{tabular}

(b)

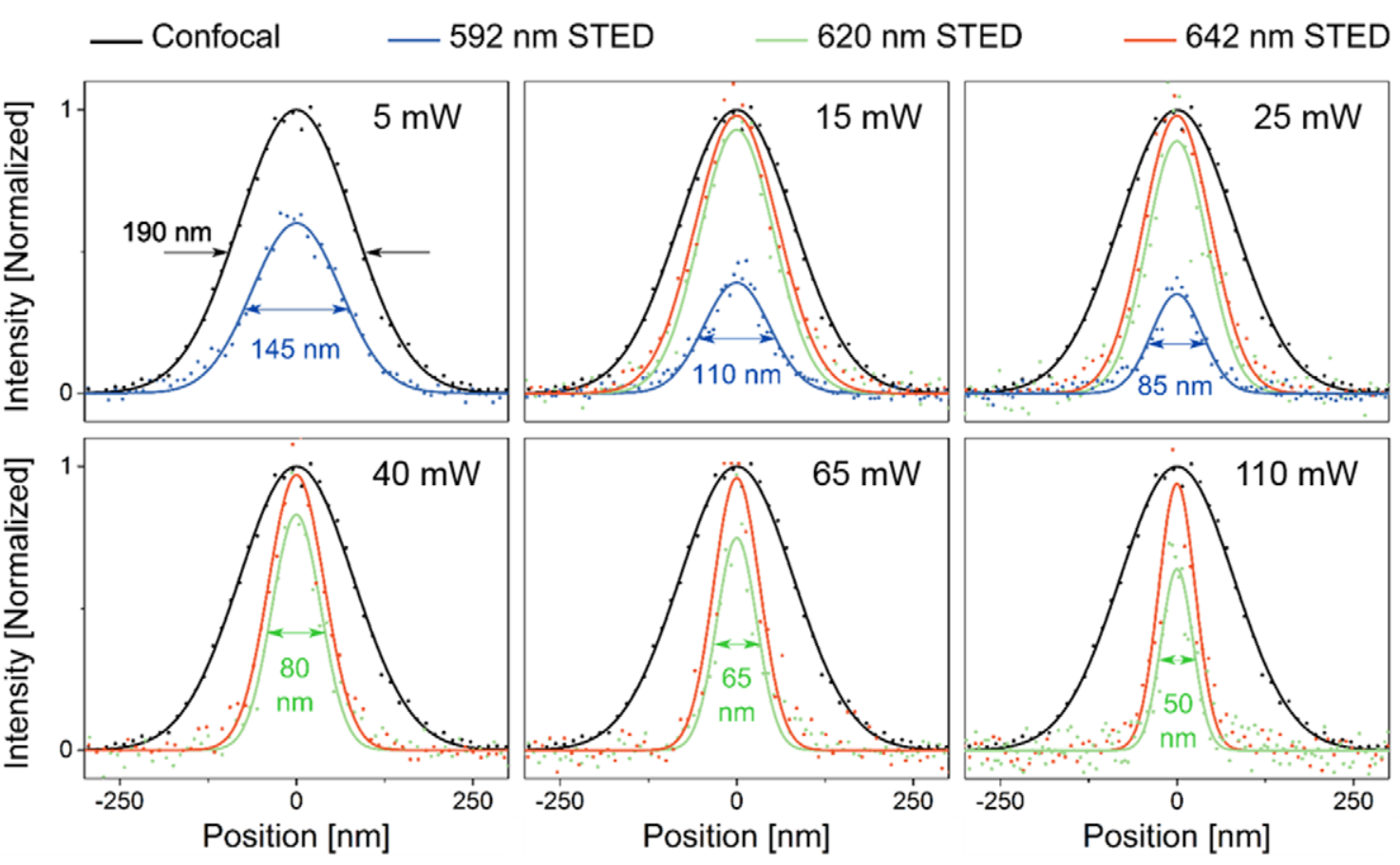

Figure 3. (a) Representative images of individual $20 \mathrm{~nm}$ polystyrene nanoparticles dyed with Nile Red obtained with STED wavelengths of 592, 620 and $642 \mathrm{~nm}$, at six different powers $\left(P_{\mathrm{STED}}\right)$. Each image corresponds to different areas of the sample of $0.5 \mu \mathrm{m}$ by $1 \mu \mathrm{m}$. Conditions labeled as 'no change' indicate that the spatial resolution was identical to confocal. Conditions labeled as 'low signal' indicate that the relation between STED and confocal signal was lower than $15 \%$. For reference, a confocal image is shown, too. (b) Representative signal profiles and fits for each condition. The FWHM illustrates the spatial resolution achieved in each case.

It should be noted that the quantum efficiency of Nile Red depends dramatically on the polarity of the environment. Therefore, it is sensible to expect smaller values of $\sigma_{\mathrm{se}}$, but since the quantum efficiency is a multiplicative constant in equation (1), the relation of $\sigma_{\mathrm{se}}(592 \mathrm{~nm}): \sigma_{\mathrm{se}}(620 \mathrm{~nm}): \sigma_{\mathrm{se}}(642 \mathrm{~nm})=1: 0.8: 0.5$ should hold. In comparison to typical configurations with the STED laser tuned at the red tail of the emission spectrum, e.g. $660 \mathrm{~nm}$ [26], 676 nm [27] and $765 \mathrm{~nm}[28,29]$, the wavelengths used here correspond to stimulated emission cross sections between 2- and 10-fold larger.

\subsection{CW STED nanoscope}

A schematic of the time-gated CW-STED nanoscope is shown in figure 2(a). For excitation, $488 \mathrm{~nm}$ light was selected with an interference filter (Chroma ZET488/10X) from a pulsed supercontinuum laser (SL; Koheras SuperK Extreme) operating at $80 \mathrm{MHz}$ repetition. After passing through a polarizing beam splitter (PBS; Thorlabs) it was coupled into a polarization maintaining single-mode fiber (PMF1; Thorlabs P3-488-PM-FC-2) using a fiber collimator (L1; Schäfter und Kirchhoff 60FC-4-A7.5-01). Finally, light exiting the fiber was collimated (L3; $f=15 \mathrm{~mm}$ ) in order to obtain the TE00 excitation beam. For STED, three linearly polarized CW lasers at 592, 620 and $642 \mathrm{~nm}$ were used (MPB Communications 2RU-VFL-P-1500-592/620/642). The three STED lasers were coupled into the same polarization maintaining single mode fiber (PMF2; Schäfter und Kirchhoff PMC-630-4.2-NA0123-APC/EC-500-P) using a fiber collimator (L2; Schäfter und Kirchhoff 60FC-4-A7.5-02). STED light coming out of the fiber was then collimated (L4; $f=15 \mathrm{~mm}$ ) and sent through 
a $2 \pi$ vortex phase mask (VP; RPC Photonics VPP-1a). Excitation and STED beams were combined using a dichroic mirror (DM; Chroma Z590sprdc).

In order to separate the excitation and STED beams from fluorescence light, we built a versatile acousto-optical beam splitter (AOBS) composed of two acousto-optical tunable filters (AOTF; AA Opto-electronic AA-AOTFnC). The AOTF 1 is set to diffract both excitation and STED wavelengths and direct them towards the sample, where they are focused to the diffraction limit with an objective of $1.4 \mathrm{NA}(\mathrm{O}$; Leica HCX PL APO 100x/1.40-0.70 Oil CS). Fluorescence light is collected by the same objective and passes through the AOTF 1 un-diffracted (zero order) but diverging and with considerable chromatic dispersion. These aberrations are corrected by a second crystal with the same characteristics (AOTF 2) used in a reversed way. The AOBS has two main advantages for multicolour nanoscopy in comparison to mirror-type beam splitters: (i) it does not suffer from mechanical instabilities associated with rotating filter wheels, which lead to image shifts, and (ii) it can change the dichroic settings of the microscope in about $15 \mu \mathrm{s}$. The average transmission at optical wavelengths between $450-700 \mathrm{~nm}$ is of about $95 \%$. Additional suitable band-pass and notch filters (Chroma, Semrock) were used before coupling the fluorescence light into the multimode fiber (MMF; Thorlabs M31L02) of the confocal detector (APD_Perkin Elmer SPCM-AQRH-13-FC).

A telescope of $2.4 \times$ magnification (L5: L6) was used to resize the waists of the beams to overfill the pupil of the objective. Lateral beam scanning of the sample was performed by a system composed of a galvanometric scanner (Till Photonics Yanus IV), a scanning lens (SL; Leica) and a tube lens (TL; Leica). In order to obtain circular polarization a broadband quarter-waveplate (B. Halle) was used. For additional positional control and fine focusing, the sample was mounted on a XYZ piezoelectric nanopositioning stage (Thorlabs NanoMax MAX311D/M). The arrival times of fluorescence photons were detected with a TCSPC (time-correlated single photon counting) device (Pico Harp 300, PicoQuant). The scanning, fluorescence count acquisition and acousto-optic devices were computer controlled via AD/DA board (National Instruments PCIe-6353).

\subsection{STED imaging}

For each STED wavelength, the state of circular polarization was optimized and a suitable vortex phase plate was implemented. The quality of the doughnut-shaped focus as well as the co-alignment with the excitation focus was adjusted while scanning over a spherical gold nanoparticle of $40 \mathrm{~nm}$ in diameter, using as feedback the scattering signal. Fluorescence photons were filtered spectrally and temporally. For spectral filtering band-pass and notch filters were used in the detection: for STED at $642 \mathrm{~nm}$ : (585 \pm 35$) \mathrm{nm}$ and notch (OD $\geqslant 6$; $642 \pm 2) \mathrm{nm}$; for STED at $620 \mathrm{~nm}:(585 \pm 35) \mathrm{nm}$ and notch $(\mathrm{OD} \geqslant 6 ; 620 \pm 2) \mathrm{nm}$; and for STED at $592 \mathrm{~nm}$ : (550 \pm 44$)$ $\mathrm{nm}$ and notch $(\mathrm{OD} \geqslant 6 ; 592 \pm 2) \mathrm{nm}$. Temporal filtering was performed with a strategy similar to the one reported [15] in order to subtract the contribution of fluorescence excited by the STED beam. During scanning, the detection time of each photon after the excitation pulse was recorded with a TCSPC device. With this information, two images were generated with the photons detected at different time windows within the 12.5 ns between successive excitation pulses (figure 2(b)). Image 1 was produced with the photons detected on a time window $0.5 \mathrm{~ns}<t<4.5 \mathrm{~ns}$ after the excitation pulse, and therefore is composed of photon counts corresponding to the super-resolved STED image plus photon counts corresponding to molecules excited by the STED beam. Image 2 was produced with the photons detected in a time window $4.5 \mathrm{~ns}<t<8.5 \mathrm{~ns}$ after the excitation pulse. Since Nile Red has an excited state lifetime of $(3 \pm 0.1) \mathrm{ns}$, this time window avoids the majority of fluorescence photons generated by the pulsed excitation, and therefore is composed mainly of fluorescence photons excited by the CW STED beam. The super-resolved STED image is then obtained simply by the subtraction Image 1 - Image 2. The impact of this temporal filtering on the super-resolved images increases as the STED wavelength decreases: it was essential for STED at $592 \mathrm{~nm}$, but led only to minor improvements for STED at $642 \mathrm{~nm}$.

For each STED wavelength and power, sets of images (confocal + STED) were acquired from different areas of the sample, where individual beads could be found. Imaging parameters: $10 \times 10 \mu \mathrm{m}, 700 \times 700$ pixels $(14.3 \mathrm{~nm} /$ pixel $)$, pixel dwell time of $100 \mu \mathrm{s}, 10 \mu \mathrm{W}$ of excitation power measured at the entrance pupil of the objective.

\section{Results and discussion}

We evaluated the performance of each one of the three STED wavelengths by analyzing images obtained with different STED beam powers. In figure 3(a), an array composed of typical STED images obtained under different STED wavelength and power are shown for visual comparison. Remarkably, we obtained super-resolved STED images with all STED wavelengths, even with $592 \mathrm{~nm}$, which practically coincides with the emission maximum of Nile Red (figure 1). As a measure of the achieved spatial resolution we use the full width at half maximum (FWHM) obtained from fitting a Gaussian function to the signal of individual beads; representative signal profiles and fits are shown in figure 3(b). For a given STED beam power, as the wavelength gets closer to the emission maximum two effects are evident: (i) the resolution improves, as expected from the increased stimulated emission cross section, and (ii) the overall signal decreases. Next, we analyze these effects in detail and discuss their implications for STED nanoscopy.

For a quantitative analysis of the power dependent resolution achieved with each STED wavelength, we computed the average FWHM of at least 50 STED images of nanoparticles for each combination of STED wavelength and power. The results are shown in figure 4(a).

The resolution of a confocalized gated CW STED nanoscope can be expressed as [23]:

$$
d=\frac{d_{\mathrm{c}}}{\sqrt{1+d_{\mathrm{c}}^{2} \zeta\left(1+\frac{\gamma}{\ln (2)}\right) \beta}}
$$


(a)

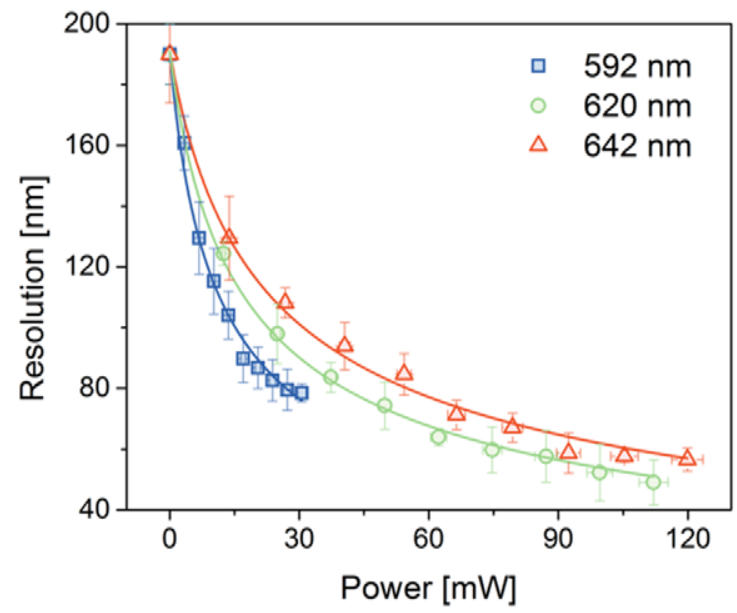

(b)

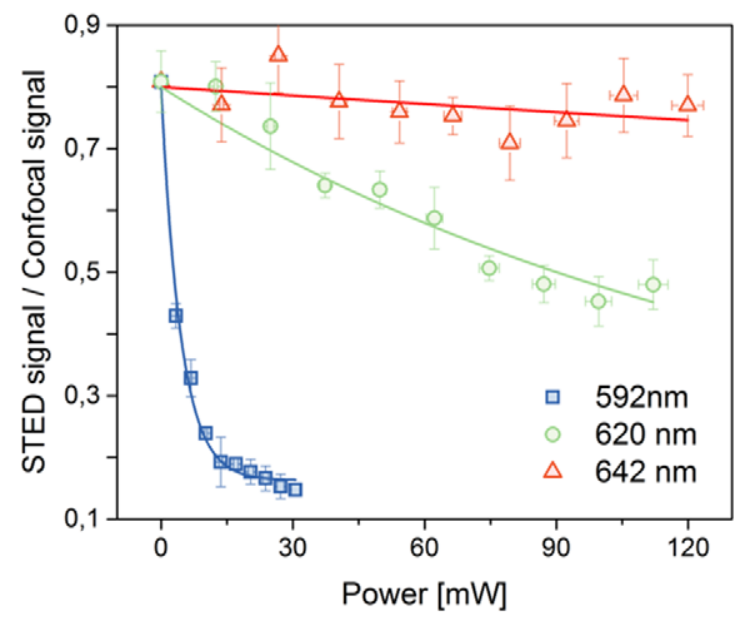

Figure 4. (a) Resolution (average FWHM of at least 50 beads for each data point) as a function of the STED laser power for three different wavelengths. The lines are fits using equation (2). (b) STED signal/confocal signal as a function of the STED beam power for three different wavelengths. The lines are exponential fits to help visualize the trend.

where $d_{\mathrm{c}}$ is the resolution (FWHM) of the plain confocal recording. $\zeta, \gamma$ and $\beta$ are factors that take into account the saturation of the stimulated emission, the effect of time gating, and the power distribution near the center of a doughnutshaped focus, respectively.

$\zeta$ is defined as $\zeta=\frac{I_{\mathrm{STED}}^{m}}{I_{\mathrm{S}}}$, where $I_{\mathrm{S}}=\frac{1}{\sigma_{\mathrm{Se}} \tau}$ and $I_{\mathrm{STED}}^{m}=\frac{P_{\mathrm{STED}}}{A_{\mathrm{STED}}} \frac{\lambda}{h c}$. In order to estimate the area of the STED focus $A_{\text {STED }}$, we compare the intensities of a Gaussian beam and a doughnutshaped STED beam expressed as $I_{\mathrm{G}}(r)=I_{\mathrm{G}}^{m} \mathrm{e}^{-4 \ln 2 \frac{r^{2}}{\mathrm{FWHM}^{2}}}$ and $I_{\mathrm{STED}}(r)=\frac{I_{\mathrm{STED}}^{m}}{0.184} \frac{r^{2}}{\mathrm{FWHM}^{2}} 2 \ln 2 \mathrm{e}^{-4 \ln 2 \frac{r^{2}}{\mathrm{FWHM}^{2}}}, \quad$ respectively, where $I_{\mathrm{G}}^{m}=\frac{2}{\pi} 2 \ln 2 \frac{P}{\mathrm{FWHM}^{2}} \quad$ and $\quad I_{\mathrm{STED}}^{m}=\frac{4}{\pi} 2 \ln 2 \frac{P}{\mathrm{FWHM}^{2}} 0.184 \quad$ are defined in such a way that integration over the space equals the power $P$. Then, it turns out that the relationship between the areas of a Gaussian beam and a doughnut-shaped beam is roughly 0.3. We therefore estimate $A_{\mathrm{STED}} \approx \frac{\pi}{0.3}\left(\frac{\mathrm{FWHM}}{2}\right)^{2}$.

$\gamma$ is defined as $\gamma=\frac{T_{\mathrm{g}}}{\tau}$, with $T_{\mathrm{g}}$ being the delay between the excitation pulse and the beginning of the temporal window for fluorescence collection. It is assumed that the collection timewindow extends well beyond the spontaneous decay time $\tau$.

The factor $\beta$ is obtained from a fit to the intensity profile of the doughnut-shaped STED beams near the central minimum according to $I_{\mathrm{STED}}(r)=4 \beta I_{\mathrm{STED}}^{m} r^{2}$. This equation assumes that the intensity at the central minimum is zero, which is a sensible approximation since in our measurements, it was smaller than $1 \%$ of the doughnut crest intensity, as shown in figure 2(b).

From these numerous parameters, the only one unknown is $\sigma_{\text {se }}$. The excited-state lifetime of Nile Red in our experimental conditions was determined to be $\tau=(3 \pm 0.1) \mathrm{ns}$. $P_{\text {STED }}$ was measured at the entrance pupil of the objective with a calibrated power meter and the transmission of the objective lens for each STED wavelength was considered. The gated detection was carried out with $T_{\mathrm{g}}=(0.5 \pm 0.1) \mathrm{ns}$. The resolution of the confocal microscope was measured from more than 150 polystyrene nanoparticles dyed with Nile Red $d_{\mathrm{c}}=(190 \pm 10) \mathrm{nm}$. The value of FWHM, as well as the value of the intensity distribution $I_{\mathrm{STED}}(r)$ were determined from measurements of scattered light while scanning a spherical $40 \mathrm{~nm}$ gold nanoparticle over the corresponding foci. Representative signal profiles and fits are shown in figure 2(b). The obtained values of FWHM and $\beta$ for the used STED wavelengths are shown in table 1.

It is possible to fit the resolution versus STED beam power with equation (2), being $\sigma_{\text {se }}$ the only free parameter (figure 4(a)). Overall, the description by equation (2) provides a satisfactory fit of the experimental results, leading to the values of $\sigma_{\text {se }}$ for each STED wavelength shown in table 1. Also in table 1 we show the values $\sigma_{\text {se }}$ predicted using equation (1), the emission spectrum, and the reported value of quantum efficiency $\phi=0.7$. The values of $\sigma_{\mathrm{se}}$ obtained from the STED resolution measurements are on average 3.7 times smaller than the ones calculated with equation (1). However, their relative magnitude holds fairly well within experimental error $\left(\sigma_{\mathrm{se}}(592 \mathrm{~nm}): \sigma_{\mathrm{se}}(620 \mathrm{~nm}): \sigma_{\mathrm{se}}(642 \mathrm{~nm})=1: 0.75: 0.59\right)$. This is indicative that the quantum efficiency of Nile Red in our experimental conditions is close to $\phi=0.2$, rather than $\phi=0.7$ as used in the calculations.

It is interesting to compare our results to reported data on the same fluorophore. Nile Red has been successfully used for STED nanoscopy with various STED wavelengths at the red tail of the emission spectrum, such as 660 [26], 676 [27] and $765 \mathrm{~nm}$ [28, 29], among others. The stimulated emission cross sections for each of those wavelengths, calculated from the emission spectrum and for $\phi=0.2$, are $\sigma_{\mathrm{se}}(660 \mathrm{~nm})=1.4 \times 10^{-17} \mathrm{~cm}^{2}, \quad \sigma_{\mathrm{se}}(676 \mathrm{~nm})=1.0 \times 10^{-17} \mathrm{~cm}^{2}$ and $\sigma_{\mathrm{se}}(765 \mathrm{~nm})=0.36 \times 10^{-17} \mathrm{~cm}^{2}$. Using wavelengths across the emission spectrum increases the efficiency of STED photons up to 10 times.

We next analyzed the fluorescence signal intensity obtained with the three STED configurations. From a direct inspection of the images and profiles (figure 3), it is evident that the signal drops as the STED wavelength approaches the emission 
Table 1. Experimentally determined values of $\beta$, FWHM and $\sigma_{\text {se }}$ for the three STED wavelengths.

\begin{tabular}{|c|c|c|c|}
\hline Parameter/STED wavelength & $592 \mathrm{~nm}$ & $620 \mathrm{~nm}$ & $642 \mathrm{~nm}$ \\
\hline$\beta\left(\mathrm{m}^{-2}\right)$ & $2.4 \times 10^{13}$ & $2.2 \times 10^{13}$ & $2.1 \times 10^{13}$ \\
\hline FWHM (nm) & $280 \pm 10$ & $290 \pm 10$ & $300 \pm 10$ \\
\hline$\sigma_{\mathrm{se}}\left(\mathrm{cm}^{2}\right)$-from STED resolution (equation (2)) & $3.7 \times 10^{-17}$ & $2.8 \times 10^{-17}$ & $2.2 \times 10^{-17}$ \\
\hline$\sigma_{\mathrm{se}}($ norm.)-from STED resolution (equation (2)) & 1 & 0.75 & 0.59 \\
\hline$\sigma_{\mathrm{se}}\left(\mathrm{cm}^{2}\right)-$ from emission spectrum (equation (1)) & $1.4 \times 10^{-16}$ & $1.1 \times 10^{-16}$ & $0.7 \times 10^{-16}$ \\
\hline$\sigma_{\mathrm{se}}($ norm.) - from emission spectrum (equation (1)) & 1 & 0.8 & 0.5 \\
\hline
\end{tabular}

Note: Values of $\sigma_{\mathrm{se}}$ obtained from fits to the power-dependent STED resolution (figure 4(a), equation (2)), and from the emission spectrum and reported value of quantum efficiency $\phi=0.7$ (figure 1, equation (1)).

maximum. First, it is important to consider the spectral windows used for fluorescence detection, which are typically placed between the excitation and the STED wavelengths (figure 1). Therefore, the integrated fluorescence signal may be considerably smaller for STED wavelengths approaching the emission maximum. At this point we note that, in principle, it is possible to collect fluorescence light at wavelengths longer than the STED wavelength too. However, this was impractical in our case because the intense CW STED beams excited fluorescence on optical components of the set-up (specifically on the scanning lens and the crystal of the AOTF), which led to unacceptable background levels. In our experiments the integrated fluorescence signal with the detection window 1 (STED at $592 \mathrm{~nm}$, figure 1) corresponds to $36 \%$ of the total emission, whereas the detection window 2 (STED at $620 \mathrm{~nm}$ and $642 \mathrm{~nm}$ ) corresponds to $55 \%$ of the total emission. Nevertheless, the observed signal reduction is more drastic than what is to be expected from these different spectral detection windows, and strongly dependent on the STED power and wavelength. We imaged all beads confocally after STED imaging (i.e. the imaging sequence was confocal-STED-confocal) and observed that the signal reduction was irreversible, which is indicative of photobleaching induced by the STED lasers.

A quantification of the signal reduction as a function of the STED beam power and wavelength is shown in figure 4(b). We computed the average reduction in the fluorescence signal of the nanoparticles from a first confocal image and a subsequent STED image, using the different depletion wavelengths and powers. An average signal reduction of $20 \%$ was observed after two confocal scans; i.e. even in the absence of any STED laser. Applying the STED laser at $642 \mathrm{~nm}$ has a minor influence on the fluorescence signal; it only reduces an extra $5 \%$ even when using $(120 \pm 4) \mathrm{mW}$ of STED beam power. Under these conditions, a resolution of $(57 \pm 4) \mathrm{nm}$ is achieved. Shifting the STED wavelength to $620 \mathrm{~nm}$ leads to a considerable reduction of fluorescence signal, with an almost linear dependence on the STED beam power. When $(110 \pm 3) \mathrm{mW}$ of power at $620 \mathrm{~nm}$ are used, the fluorescence signal drops an additional $30 \%$ in comparison to the situation with no STED laser. Still, we obtained reasonable images with $(49 \pm 7)$ $\mathrm{nm}$ resolution. The situation changes dramatically when the STED wavelength is tuned to $592 \mathrm{~nm}$. In this case, the signal drops to $15 \%$ of its original level after a confocal and a STED image using only $(30 \pm 1) \mathrm{mW}$ of STED beam power. Such a strong photodegradation is at the edge of what is viable for
CW STED imaging, and limited the achieved resolution to $(78 \pm 3) \mathrm{nm}$.

\section{Conclusions}

The main conclusion of this work is that CW STED nanoscopy is not limited to using STED lasers with wavelengths at the red tail of the emission spectrum of fluorophores. It is in fact feasible using practically any STED wavelength at the emission spectrum. Since the stimulated emission cross section follows practically the same spectral dependence as the emission, using STED wavelengths closer to the emission maximum makes STED imaging considerably more efficient, in terms of the power needed for STED. We modeled the STED resolution quantitatively as a function of the stimulated emission cross section. In comparison to typical STED configurations, using STED wavelengths at the emission peak can lead to stimulated emission cross sections up to 10 times larger.

We found that for this fluorophore, photodegradation becomes critical as the STED wavelength approaches the absorption band. This is understandable as the fluorophore increasingly undergoes (undesired) excitation and deexcitation cycles elicited by the STED beam. While the cycling is not harmful by itself, the dye is more prone to be further excited to a high-lying excited state and hence to photobleaching. Using a STED wavelength at half the emission maximum $(620 \mathrm{~nm})$ was a very good compromise between using a higher stimulated emission cross section and bleaching. Concretely, we could make use of a stimulated emission cross section eight times larger than at the red tail of the spectrum, at a 50\% reduction of signal. Naturally, these conditions will vary for different fluorophores.

Given the crucial role of their states in the process, fluorophores are essential to obtain high-quality far-field nanoscopy images. Their performance and photodegradation have been extensively studied in red tail wavelength STED configurations. The results of this work are motivating for testing fluorophores under STED wavelengths at the emission maximum. First, STED nanoscopy using considerably lower power densities for stimulated emission depletion may become possible, and second, fluorophores that have been reported inadequate under typical STED configurations may become useful simply by using wavelengths where their stimulated emission cross sections are several times larger. 


\section{Acknowledgments}

This work was financially supported by a Partner Group grant of the Max Planck Society. We thank Katrin Willig, Volker Westphal, Andreas Schönle and Jaydev Jethwa for valuable help regarding the design of the STED nanoscope.

\section{References}

[1] Hell S W 2003 Toward fluorescence nanoscopy Nat. Biotechnol. 21 1347-55

[2] Hell S W 2009 Microscopy and its focal switch Nat. Methods 6 24-32

[3] Hell S W and Wichmann J 1994 Breaking the diffraction resolution limit by stimulated emission: stimulatedemission-depletion fluorescence microscopy Opt. Lett. $19780-2$

[4] Testa I, Urban N T, Jakobs S, Eggeling C, Willig K I and Hell S W 2012 Nanoscopy of living brain slices with low light levels Neuron 75 992-1000

[5] Betzig E, Patterson G H, Sougrat R, Lindwasser O W, Olenych S, Bonifacino J S, Davidson M W, LippincottSchwartz J and Hess H F 2006 Imaging intracellular fluorescent proteins at nanometer resolution Science 313 1642-5

[6] Rust M J, Bates M and Zhuang X 2006 Sub-diffraction-limit imaging by stochastic optical reconstruction microscopy (STORM) Nat. Methods 3 793-5

[7] Sharonov A and Hochstrasser R M 2006 Wide-field subdiffraction imaging by accumulated binding of diffusing probes Proc. Natl Acad. Sci. 103 18911-6

[8] Rittweger E, Rankin B R, Westphal V and Hell S W 2007 Fluorescence depletion mechanisms in super-resolving STED microscopy Chem. Phys. Lett. 442 483-7

[9] Deshpande A V, Beidoun A, Penzkofer A and Wagenblast G 1990 Absorption and emission spectroscopic investigation of cyanovinyldiethylaniline dye vapors Chem. Phys. 142 123-31

[10] Tinnefeld P, Eggeling C and Hell S W 2014 Far-Field Optical Nanoscopy (New York: Springer)

[11] Wäldchen S, Lehmann J, Klein T, van de Linde S and Sauer M 2015 Light-induced cell damage in live-cell superresolution microscopy Sci. Rep. 515348

[12] Hell S W, Dyba M and Jakobs S 2004 Concepts for nanoscale resolution in fluorescence microscopy Curr. Opin. Neurobiol. 14 599-609

[13] Hofmann M, Eggeling C, Jakobs S and Hell S W 2005 Breaking the diffraction barrier in fluorescence microscopy at low light intensities by using reversibly photoswitchable proteins Proc. Natl Acad. Sci. 102 17565-9
[14] Testa I, D’Este E, Urban N T, Balzarotti F and Hell S W 2015 Dual channel RESOLFT nanoscopy by using fluorescent state kinetics Nano Lett. 15 103-6

[15] Staudt T, Engler A, Rittweger E, Harke B, Engelhardt J and Hell S W 2011 Far-field optical nanoscopy with reduced number of state transition cycles Opt. Express 19 5644-57

[16] Hein B, Willig K I and Hell S W 2008 Stimulated emission depletion (STED) nanoscopy of a fluorescent proteinlabeled organelle inside a living cell Proc. Natl Acad. Sci. USA 105 14271-6

[17] Vicidomini G, Moneron G, Han K Y, Westphal V, Ta H, Reuss M, Engelhardt J, Eggeling C and Hell S W 2011 Sharper low-power STED nanoscopy by time gating Nat. Methods 8 571-3

[18] Nägerl U V, Willig K I, Hein B, Hell S W and Bonhoeffer T 2008 Live-cell imaging of dendritic spines by STED microscopy Proc. Natl Acad. Sci. 105 18982-7

[19] Urban N T, Willig K I, Hell S W and Nägerl U V 2011 STED nanoscopy of actin dynamics in synapses deep inside living brain slices Biophys. J. 101 1277-84

[20] D'Este E, Kamin D, Göttfert F, El-Hady A and Hell S W 2015 STED nanoscopy reveals the ubiquity of subcortical cytoskeleton periodicity in living neurons Cell Rep. 10 1246-51

[21] Moneron G, Medda R, Hein B, Giske A, Westphal V and Hell S W 2010 Fast STED microscopy with continuous wave fiber lasers Opt. Express 18 1302-9

[22] Berning S, Willig K I, Steffens H, Dibaj P and Hell S W 2012 Nanoscopy in a living mouse brain Science $\mathbf{3 3 5} 551$

[23] Vicidomini G, Moneron G, Eggeling C, Rittweger E and Hell S W 2012 STED with wavelengths closer to the emission maximum Opt. Express 20 5225-36

[24] Ronzitti E, Harke B and Diaspro A 2013 Frequency dependent detection in a STED microscope using modulated excitation light Opt. Express 21 210-9

[25] Sackett D L and Wolff J 1987 Nile red as a polarity-sensitive fluorescent probe of hydrophobic protein surfaces Anal. Biochem. 167 228-34

[26] Schrof S, Staudt T, Rittweger E, Wittenmayer N, Dresbach T, Engelhardt J and Hell S W 2011 STED nanoscopy with mass-produced laser diodes Opt. Express 19 8066-72

[27] Moneron G and Hell S W 2009 Two-photon excitation STED microscopy Opt. Express 17 14567-73

[28] Hell S W, Willig K I, Dyba M, Jakobs S, Kastrup L and Westphal V 2006 Nanoscale resolution with focused light: stimulated emission depletion and other reversible saturable optical fluorescence transitions microscopy concepts Handbook of Biological Confocal Microscopy (Boston: Springer) pp 571-9

[29] Klar T A, Jakobs S, Dyba M, Egner A and Hell S W 2000 Fluorescence microscopy with diffraction resolution barrier broken by stimulated emission Proc. Natl Acad. Sci. $978206-10$ 